\title{
New formulae of products of the Frobenius-Euler polynomials
}

Yuan $\mathrm{He}^{*}$ and Sherry J Wang

"Correspondence:
hyyhe@aliyun.com
Faculty of Science, Kunming
University of Science and
Technology, Kunming, Yunnan
650500, People's Republic of China

\section{"Correspondence:} hyyhe@aliyun.com Faculty of Science, Kunming Technology, Kunming, Yunnan 650500, People's Republic of China

\begin{abstract}
In this paper, we perform a further investigation for the Frobenius-Euler polynomials. Some new formulae of products of the Frobenius-Euler polynomials are established by applying the generating function methods and some summation transform techniques. It turns out that some corresponding known results are obtained as special cases.

MSC: 11B68;05A19

Keywords: Bernoulli polynomials and numbers; Euler polynomials and numbers; Frobenius-Euler polynomials and numbers; combinatorial identities
\end{abstract}

\section{Introduction}

Let $\lambda$ be a complex number with $\lambda \neq 1$. Frobenius [1] studied in great detail the socalled Frobenius-Euler polynomials $H_{n}(x \mid \lambda)$ satisfying the following exponential generating function:

$$
\frac{1-\lambda}{e^{t}-\lambda} e^{x t}=\sum_{n=0}^{\infty} H_{n}(x \mid \lambda) \frac{t^{n}}{n !}
$$

In particular, $H_{n}(\lambda)=H_{n}(0 \mid \lambda)$ are called the Frobenius-Euler numbers. In fact, the Frobenius-Euler polynomials can also be defined recursively by the Frobenius-Euler numbers, as follows:

$$
H_{n}(x \mid \lambda)=\sum_{k=0}^{n}\left(\begin{array}{l}
n \\
k
\end{array}\right) H_{k}(\lambda) x^{n-k} \quad(n \geq 0)
$$

where the Frobenius-Euler numbers obey the recurrence relation

$$
H_{0}(\lambda)=1, \quad(H(\lambda)+1)^{n}-H_{n}(\lambda)= \begin{cases}1-\lambda, & n=0 \\ 0, & n \geq 1\end{cases}
$$

with the usual convention about replacing $H^{n}(\lambda)$ by $H_{n}(\lambda)$. For some interesting arithmetic properties of the Frobenius-Euler polynomials and numbers, one is referred to [2-13].

○2014 He and Wang; licensee Springer. This is an Open Access article distributed under the terms of the Creative Commons Attribution License (http://creativecommons.org/licenses/by/2.0), which permits unrestricted use, distribution, and reproduction in any medium, provided the original work is properly cited. 
Some analogues of the Frobenius-Euler polynomials are the classical Bernoulli polynomials $B_{n}(x)$ and Euler polynomials $E_{n}(x)$. They are usually defined by the following exponential generating functions:

$$
\frac{t e^{x t}}{e^{t}-1}=\sum_{n=0}^{\infty} B_{n}(x) \frac{t^{n}}{n !} \quad \text { and } \quad \frac{2 e^{x t}}{e^{t}+1}=\sum_{n=0}^{\infty} E_{n}(x) \frac{t^{n}}{n !} .
$$

Especially, the rational numbers $B_{n}=B_{n}(0)$ and integers $E_{n}=2^{n} E_{n}(1 / 2)$ are called the classical Bernoulli numbers and Euler numbers, respectively. These numbers and polynomials play important roles in many different areas of mathematics including number theory, combinatorics, special functions and analysis. Numerous interesting properties for them can be found in many books; see, for example, [14-17]. Obviously the Frobenius-Euler polynomials give the classical Euler polynomials when $\lambda=-1$ in (1.1).

In [15], Nielsen investigated three formulae of products of the classical Bernoulli and Euler polynomials. In particular, Nielsen showed that for positive integers $m, n$,

$$
\begin{aligned}
B_{m}(x) B_{n}(x)= & \sum_{k=0}^{\infty}\left\{n\left(\begin{array}{c}
m \\
2 k
\end{array}\right)+n\left(\begin{array}{c}
n \\
2 k
\end{array}\right)\right\} B_{2 k} \frac{B_{m+n-2 k}(x)}{m+n-2 k} \\
& +(-1)^{m+1} \frac{m ! \cdot n !}{(m+n) !} B_{m+n},
\end{aligned}
$$

which was reobtained by Carlitz [18] applying a different method. For further discoveries of Nielsen's formulae on the classical Bernoulli and Euler polynomials, see [19-23] for details. In [24], Carlitz explored some formulae of products of the Frobenius-Euler polynomials and obtained that for non-negative integers $m, n$,

$$
\begin{aligned}
H_{m}(x \mid \lambda) H_{n}(x \mid \mu)= & \frac{\lambda(\mu-1)}{\lambda \mu-1} \sum_{k=0}^{m}\left(\begin{array}{l}
m \\
k
\end{array}\right) H_{k}(\lambda) H_{m+n-k}(x \mid \lambda \mu) \\
& +\frac{\mu(\lambda-1)}{\lambda \mu-1} \sum_{k=0}^{n}\left(\begin{array}{l}
n \\
k
\end{array}\right) H_{k}(\mu) H_{m+n-k}(x \mid \lambda \mu) \\
& -\frac{(\lambda-1)(\mu-1)}{\lambda \mu-1} H_{m+n}(x \mid \lambda \mu),
\end{aligned}
$$

when $\lambda \neq 1, \mu \neq 1, \lambda \mu \neq 1$, and if $\lambda \neq 1$, then for positive integer $m$ and non-negative integer $n$,

$$
\begin{aligned}
B_{m}(x) H_{n}(x \mid \lambda)= & \sum_{k=0}^{m}\left(\begin{array}{c}
m \\
k
\end{array}\right) B_{k} H_{m+n-k}(x \mid \lambda) \\
& -\frac{\lambda}{\lambda-1} m \sum_{k=0}^{n}\left(\begin{array}{l}
n \\
k
\end{array}\right) H_{k}(\lambda) H_{m+n-k-1}(x \mid \lambda) \\
& +m H_{m+n-1}(x \mid \lambda),
\end{aligned}
$$


and if $\lambda \neq 0,1$, then for positive integers $m, n$,

$$
\begin{aligned}
H_{m}(x \mid \lambda) H_{n}\left(x \mid \frac{1}{\lambda}\right)= & (\lambda-1) \sum_{k=1}^{m}\left(\begin{array}{c}
m \\
k
\end{array}\right) H_{k}(\lambda) \frac{B_{m+n+1-k}(x)}{m+n+1-k} \\
& +\left(\frac{1}{\lambda}-1\right) \sum_{k=1}^{n}\left(\begin{array}{l}
n \\
k
\end{array}\right) H_{k}\left(\frac{1}{\lambda}\right) \frac{B_{m+n+1-k}(x)}{m+n+1-k} \\
& +(-1)^{n}(\lambda-1) \frac{m ! \cdot n !}{(m+n+1) !} H_{m+n+1}(\lambda) .
\end{aligned}
$$

Further, Carlitz [24] showed that the case $\lambda=-1$ in (1.7) and (1.8) can be used to give the expression of $B_{m}(x) E_{n}(x)$ and $E_{m}(x) E_{n}(x)$ stated in [15], respectively.

Motivated by the work of Carlitz [24], in the present paper we establish some new formulae of products of the Frobenius-Euler polynomials by applying the generating function methods and some summation transform techniques. It turns out that some known results including (1.6), (1.7) and (1.8) are obtained as special cases.

\section{Restatement of the main results}

In this section, we shall establish three new formulae of products of the Frobenius-Euler polynomials to extend formulae (1.6), (1.7) and (1.8) by making use of the generating function methods. As further applications, we also give some new sum relations of products of the Frobenius-Euler polynomials by applying some summation transform techniques, which are analogous to some sum relations of products of the classical Euler polynomials and Frobenius-Euler polynomials stated in $[9,25]$. For convenience, in the following we shall denote by $H_{n}$ the harmonic number of order $n$ given by

$$
H_{0}=0 \quad \text { and } \quad H_{n}=\sum_{k=1}^{n} \frac{1}{k}=1+\frac{1}{2}+\cdots+\frac{1}{n} \quad(n \geq 1)
$$

We now state our main results as follows.

Theorem 2.1 Let $m, n$ be non-negative integers. Then, for $\lambda \neq 1, \mu \neq 1, \lambda \mu \neq 1$,

$$
\begin{aligned}
H_{m}(x \mid \lambda) H_{n}(y \mid \mu)= & \frac{\lambda(\mu-1)}{\lambda \mu-1} \sum_{k=0}^{m}\left(\begin{array}{l}
m \\
k
\end{array}\right) H_{m-k}(x-y \mid \lambda) H_{n+k}(y \mid \lambda \mu) \\
& +\frac{\mu(\lambda-1)}{\lambda \mu-1} \sum_{k=0}^{n}\left(\begin{array}{l}
n \\
k
\end{array}\right) H_{n-k}(y-x \mid \mu) H_{m+k}(x \mid \lambda \mu) \\
& -\frac{(\lambda-1)(\mu-1)}{\lambda \mu-1} \sum_{k=0}^{m}\left(\begin{array}{l}
m \\
k
\end{array}\right)(x-y)^{m-k} H_{n+k}(y \mid \lambda \mu) .
\end{aligned}
$$

Proof Observe that

$$
\frac{1}{\lambda e^{u}-1} \cdot \frac{1}{\mu e^{v}-1}=\left(\frac{\lambda e^{u}}{\lambda e^{u}-1}+\frac{1}{\mu e^{v}-1}\right) \frac{1}{\lambda \mu e^{u+v}-1} .
$$


If we multiply $(\lambda-1)(\mu-1) e^{x u+y v}$ on both sides of (2.3), then

$$
\begin{aligned}
\frac{(\lambda-1) e^{x u}}{\lambda e^{u}-1} \cdot \frac{(\mu-1) e^{y v}}{\mu e^{v}-1}= & \frac{\lambda(\mu-1)}{\lambda \mu-1} \cdot \frac{(\lambda-1) e^{(1+x-y) u}}{\lambda e^{u}-1} \cdot \frac{(\lambda \mu-1) e^{y(u+v)}}{\lambda \mu e^{u+v}-1} \\
& +\frac{\lambda-1}{\lambda \mu-1} \cdot \frac{(\mu-1) e^{(y-x) v}}{\mu e^{v}-1} \cdot \frac{(\lambda \mu-1) e^{x(u+v)}}{\lambda \mu e^{u+v}-1}
\end{aligned}
$$

Note that from (1.1) we have

$$
\frac{\lambda-1}{\lambda e^{u}-1} e^{x u}=\frac{1-\frac{1}{\lambda}}{e^{u}-\frac{1}{\lambda}} e^{x u}=\sum_{m=0}^{\infty} H_{m}\left(x \mid \frac{1}{\lambda}\right) \frac{u^{m}}{m !} .
$$

More generally, the Taylor theorem gives

$$
\begin{aligned}
\frac{\lambda-1}{\lambda e^{u+v}-1} e^{x(u+v)} & =\sum_{n=0}^{\infty} \frac{\partial^{n}}{\partial u^{n}}\left(\frac{\lambda-1}{\lambda e^{u}-1} e^{x u}\right) \frac{v^{n}}{n !} \\
& =\sum_{m=0}^{\infty} \sum_{n=0}^{\infty} H_{m+n}\left(x \mid \frac{1}{\lambda}\right) \frac{u^{m}}{m !} \cdot \frac{v^{n}}{n !} .
\end{aligned}
$$

By applying (2.5) and (2.6) to (2.4), we obtain

$$
\begin{aligned}
\sum_{m=0}^{\infty} \sum_{n=0}^{\infty} H_{m}\left(x \mid \frac{1}{\lambda}\right) H_{n}\left(y \mid \frac{1}{\mu}\right) \frac{u^{m}}{m !} \cdot \frac{v^{n}}{n !} \\
=\frac{\lambda(\mu-1)}{\lambda \mu-1}\left(\sum_{m=0}^{\infty} H_{m}\left(1+x-y \mid \frac{1}{\lambda}\right) \frac{u^{m}}{m !}\right)\left(\sum_{m=0}^{\infty} \sum_{n=0}^{\infty} H_{m+n}\left(y \mid \frac{1}{\lambda \mu}\right) \frac{u^{m}}{m !} \cdot \frac{v^{n}}{n !}\right) \\
+\frac{\lambda-1}{\lambda \mu-1}\left(\sum_{n=0}^{\infty} H_{n}\left(y-x \mid \frac{1}{\mu}\right) \frac{v^{n}}{n !}\right)\left(\sum_{m=0}^{\infty} \sum_{n=0}^{\infty} H_{m+n}\left(x \mid \frac{1}{\lambda \mu}\right) \frac{u^{m}}{m !} \cdot \frac{v^{n}}{n !}\right) .
\end{aligned}
$$

It follows from (2.7) and the Cauchy product that

$$
\begin{aligned}
\sum_{m=0}^{\infty} \sum_{n=0}^{\infty} H_{m}\left(x \mid \frac{1}{\lambda}\right) H_{n}\left(y \mid \frac{1}{\mu}\right) \frac{u^{m}}{m !} \cdot \frac{v^{n}}{n !} \\
=\frac{\lambda(\mu-1)}{\lambda \mu-1} \sum_{m=0}^{\infty} \sum_{n=0}^{\infty}\left[\sum_{k=0}^{m}\left(\begin{array}{l}
m \\
k
\end{array}\right) H_{m-k}\left(1+x-y \mid \frac{1}{\lambda}\right) H_{n+k}\left(y \mid \frac{1}{\lambda \mu}\right)\right] \frac{u^{m}}{m !} \cdot \frac{v^{n}}{n !} \\
+\frac{\lambda-1}{\lambda \mu-1} \sum_{m=0}^{\infty} \sum_{n=0}^{\infty}\left[\sum_{k=0}^{n}\left(\begin{array}{l}
n \\
k
\end{array}\right) H_{n-k}\left(y-x \mid \frac{1}{\mu}\right) H_{m+k}\left(x \mid \frac{1}{\lambda \mu}\right)\right] \frac{u^{m}}{m !} \cdot \frac{v^{n}}{n !} .
\end{aligned}
$$

Comparing the coefficients of $u^{m} v^{n} / m ! \cdot n !$ in (2.8) gives

$$
\begin{aligned}
H_{m}\left(x \mid \frac{1}{\lambda}\right) H_{n}\left(y \mid \frac{1}{\mu}\right)= & \frac{\lambda(\mu-1)}{\lambda \mu-1} \sum_{k=0}^{m}\left(\begin{array}{l}
m \\
k
\end{array}\right) H_{m-k}\left(1+x-y \mid \frac{1}{\lambda}\right) H_{n+k}\left(y \mid \frac{1}{\lambda \mu}\right) \\
& +\frac{\lambda-1}{\lambda \mu-1} \sum_{k=0}^{n}\left(\begin{array}{l}
n \\
k
\end{array}\right) H_{n-k}\left(y-x \mid \frac{1}{\mu}\right) H_{m+k}\left(x \mid \frac{1}{\lambda \mu}\right) .
\end{aligned}
$$


Substituting $\lambda$ for $1 / \lambda$ and $\mu$ for $1 / \mu$ in (2.9), we get

$$
\begin{aligned}
H_{m}(x \mid \lambda) H_{n}(y \mid \mu)= & \frac{\mu-1}{\lambda \mu-1} \sum_{k=0}^{m}\left(\begin{array}{l}
m \\
k
\end{array}\right) H_{m-k}(1+x-y \mid \lambda) H_{n+k}(y \mid \lambda \mu) \\
& +\frac{\mu(\lambda-1)}{\lambda \mu-1} \sum_{k=0}^{n}\left(\begin{array}{l}
n \\
k
\end{array}\right) H_{n-k}(y-x \mid \mu) H_{m+k}(x \mid \lambda \mu) .
\end{aligned}
$$

Since the Frobenius-Euler polynomials obey the difference equation (see, e.g., [9])

$$
H_{n}(x+1 \mid \lambda)-\lambda H_{n}(x \mid \lambda)=(1-\lambda) x^{n} \quad(n \geq 0)
$$

so by applying (2.11) to (2.10), the desired result follows immediately.

It follows that we show some special cases of Theorem 2.1. By setting $x=y$ in Theorem 2.1, we rediscover formula (1.6). Setting $\lambda=\mu$ and $x=y$ in Theorem 2.1, for nonnegative integers $m, n$ and $\lambda \neq \pm 1$, we have

$$
\begin{aligned}
H_{m}(x \mid \lambda) H_{n}(x \mid \lambda)= & \frac{\lambda}{\lambda+1} \sum_{k=0}^{m}\left(\begin{array}{l}
m \\
k
\end{array}\right) H_{m-k}(\lambda) H_{n+k}\left(x \mid \lambda^{2}\right) \\
& +\frac{\lambda}{\lambda+1} \sum_{k=0}^{n}\left(\begin{array}{l}
n \\
k
\end{array}\right) H_{n-k}(\lambda) H_{m+k}\left(x \mid \lambda^{2}\right)-\frac{\lambda-1}{\lambda+1} H_{m+n}\left(x \mid \lambda^{2}\right) .
\end{aligned}
$$

Clearly, $H_{0}(x \mid \lambda)=1$ (see, e.g., [9]). Hence, by setting $n=0$ in (2.12), we obtain that for non-negative integer $n$,

$$
H_{n}(x \mid \lambda)=\frac{\lambda}{\lambda+1} \sum_{k=0}^{n}\left(\begin{array}{l}
n \\
k
\end{array}\right) H_{n-k}(\lambda) H_{k}\left(x \mid \lambda^{2}\right)+\frac{1}{\lambda+1} H_{n}\left(x \mid \lambda^{2}\right) .
$$

Setting $x=y$ and $\mu=1 / \lambda^{2}$ in Theorem 2.1, we get that for non-negative integers $m, n$ and $\lambda \neq 0,1$,

$$
\begin{aligned}
H_{m}(x \mid \lambda) H_{n}\left(x \mid \frac{1}{\lambda^{2}}\right)= & (\lambda+1) \sum_{k=0}^{m}\left(\begin{array}{l}
m \\
k
\end{array}\right) H_{m-k}(\lambda) H_{n+k}\left(x \mid \frac{1}{\lambda}\right) \\
& -\frac{1}{\lambda} \sum_{k=0}^{n}\left(\begin{array}{l}
n \\
k
\end{array}\right) H_{n-k}\left(\frac{1}{\lambda^{2}}\right) H_{m+k}\left(x \mid \frac{1}{\lambda}\right)-\frac{\lambda^{2}-1}{\lambda} H_{m+n}\left(x \mid \frac{1}{\lambda}\right) .
\end{aligned}
$$

In particular, the case $n=0$ in (2.14) gives that for non-negative integer $n$,

$$
\lambda H_{n}\left(x \mid \frac{1}{\lambda}\right)+H_{n}(x \mid \lambda)=(\lambda+1) \sum_{k=0}^{n}\left(\begin{array}{l}
n \\
k
\end{array}\right) H_{n-k}\left(\frac{1}{\lambda}\right) H_{k}(x \mid \lambda),
$$

which was discovered by Kim [9] applying a nice method called the Frobenius-Euler basis $\left\{H_{0}(x \mid \lambda), \ldots, H_{n}(x \mid \lambda)\right\}$ for $\mathbb{P}_{n}$ consisting of the Frobenius-Euler polynomials with $\mathbb{P}_{n}$ being the space of polynomials of degree less than or equal to $n$. 
Theorem 2.2 Let $m, n$ be non-negative integers. Then, for $\lambda \neq 1$,

$$
\begin{aligned}
B_{m+1}(x) H_{n}(y \mid \lambda)= & \sum_{k=0}^{m+1}\left(\begin{array}{c}
m+1 \\
k
\end{array}\right) B_{m+1-k}(x-y) H_{n+k}(y \mid \lambda) \\
& -\frac{\lambda}{\lambda-1}(m+1) \sum_{k=0}^{n}\left(\begin{array}{l}
n \\
k
\end{array}\right) H_{n-k}(y-x \mid \lambda) H_{m+k}(x \mid \lambda) \\
& +(m+1) \sum_{k=0}^{m}\left(\begin{array}{c}
m \\
k
\end{array}\right)(x-y)^{m-k} H_{n+k}(y \mid \lambda) .
\end{aligned}
$$

Proof By setting $\lambda=1$ and substituting $\lambda$ for $\mu$ in (2.3), we have

$$
\frac{1}{e^{u}-1} \cdot \frac{1}{\lambda e^{v}-1}=\left(\frac{e^{u}}{e^{u}-1}+\frac{1}{\lambda e^{v}-1}\right) \frac{1}{\lambda e^{u+v}-1}
$$

Multiplying $(\lambda-1) u e^{x u+y v}$ on both sides of (2.17) leads to

$$
\begin{aligned}
\frac{u e^{x u}}{e^{u}-1} \cdot \frac{(\lambda-1) e^{y v}}{\lambda e^{v}-1}= & \frac{u e^{(1+x-y) u}}{e^{u}-1} \cdot \frac{(\lambda-1) e^{y(u+v)}}{\lambda e^{u+v}-1} \\
& +\frac{u}{\lambda-1} \cdot \frac{(\lambda-1) e^{(y-x) v}}{\lambda e^{v}-1} \cdot \frac{(\lambda-1) e^{x(u+v)}}{\lambda e^{u+v}-1} .
\end{aligned}
$$

Hence, by applying (1.4), (2.5) and (2.6) to (2.18), we obtain

$$
\begin{aligned}
\sum_{m=0}^{\infty} & \sum_{n=0}^{\infty} B_{m}(x) H_{n}\left(y \mid \frac{1}{\lambda}\right) \frac{u^{m}}{m !} \cdot \frac{v^{n}}{n !} \\
= & \left(\sum_{m=0}^{\infty} B_{m}(1+x-y) \frac{u^{m}}{m !}\right)\left(\sum_{m=0}^{\infty} \sum_{n=0}^{\infty} H_{m+n}\left(y \mid \frac{1}{\lambda}\right) \frac{u^{m}}{m !} \cdot \frac{v^{n}}{n !}\right) \\
& +\frac{u}{\lambda-1}\left(\sum_{n=0}^{\infty} H_{n}\left(y-x \mid \frac{1}{\lambda}\right) \frac{v^{n}}{n !}\right)\left(\sum_{m=0}^{\infty} \sum_{n=0}^{\infty} H_{m+n}\left(x \mid \frac{1}{\lambda}\right) \frac{u^{m}}{m !} \cdot \frac{v^{n}}{n !}\right),
\end{aligned}
$$

which together with the Cauchy product yields

$$
\begin{aligned}
\sum_{m=0}^{\infty} & \sum_{n=0}^{\infty} B_{m}(x) H_{n}\left(y \mid \frac{1}{\lambda}\right) \frac{u^{m}}{m !} \cdot \frac{v^{n}}{n !} \\
= & \sum_{m=0}^{\infty} \sum_{n=0}^{\infty}\left[\sum_{k=0}^{m}\left(\begin{array}{l}
m \\
k
\end{array}\right) B_{m-k}(1+x-y) H_{n+k}\left(y \mid \frac{1}{\lambda}\right)\right] \frac{u^{m}}{m !} \cdot \frac{v^{n}}{n !} \\
& +\frac{1}{\lambda-1} \sum_{m=0}^{\infty} \sum_{n=0}^{\infty}\left[\sum_{k=0}^{n}\left(\begin{array}{l}
n \\
k
\end{array}\right) H_{n-k}\left(y-x \mid \frac{1}{\lambda}\right) H_{m+k}\left(x \mid \frac{1}{\lambda}\right)\right] \frac{u^{m+1}}{m !} \cdot \frac{v^{n}}{n !} .
\end{aligned}
$$


Since $B_{0}(x)=1$ (see, e.g., [26]), so by substituting $\lambda$ for $1 / \lambda$ and comparing the coefficients of $u^{m+1} v^{n} /(m+1) ! \cdot n$ ! in (2.20) we get

$$
\begin{aligned}
B_{m+1}(x) H_{n}(y \mid \lambda)= & \sum_{k=0}^{m+1}\left(\begin{array}{c}
m+1 \\
k
\end{array}\right) B_{m+1-k}(1+x-y) H_{n+k}(y \mid \lambda) \\
& -\frac{\lambda}{\lambda-1}(m+1) \sum_{k=0}^{n}\left(\begin{array}{l}
n \\
k
\end{array}\right) H_{n-k}(y-x \mid \lambda) H_{m+k}(x \mid \lambda) .
\end{aligned}
$$

Thus, by applying the difference equation of the classical Bernoulli polynomials $B_{n}(x+1)-$ $B_{n}(x)=n x^{n-1}$ for non-negative integer $n$ (see, e.g., [26]) to (2.21), the desired result follows immediately.

It is obvious that the case $x=y$ in Theorem 2.2 gives formula (1.7).

Theorem 2.3 Let $m, n$ be non-negative integers. Then, for $\lambda \neq 0,1$,

$$
\begin{aligned}
H_{m}\left(x \mid \frac{1}{\lambda}\right) H_{n}(y \mid \lambda)= & \left(\frac{1}{\lambda}-1\right) \sum_{k=0}^{m}\left(\begin{array}{l}
m \\
k
\end{array}\right) H_{m-k}\left(x-y \mid \frac{1}{\lambda}\right) \frac{B_{n+k+1}(y)}{n+k+1} \\
& +(\lambda-1) \sum_{k=0}^{n}\left(\begin{array}{l}
n \\
k
\end{array}\right) H_{n-k}(y-x \mid \lambda) \frac{B_{m+k+1}(x)}{m+k+1} \\
& -\frac{(\lambda-1)^{2}}{\lambda} \sum_{k=0}^{m}\left(\begin{array}{l}
m \\
k
\end{array}\right)(x-y)^{m-k} \frac{B_{n+k+1}(y)}{n+k+1} \\
& +(-1)^{m}(\lambda-1) \frac{m ! \cdot n !}{(m+n+1) !} H_{m+n+1}(y-x \mid \lambda) .
\end{aligned}
$$

Proof By substituting $1 / \lambda$ for $\mu$ in (2.4), we discover

$$
\begin{aligned}
\frac{(\lambda-1) e^{x u}}{\lambda e^{u}-1} \cdot \frac{\left(\frac{1}{\lambda}-1\right) e^{y v}}{\frac{1}{\lambda} e^{v}-1} \\
=\lambda\left(\frac{1}{\lambda}-1\right) \frac{(\lambda-1) e^{(1+x-y) u}}{\lambda e^{u}-1}\left(\frac{e^{y(u+v)}}{e^{u+v}-1}-\frac{1}{u+v}\right) \\
\quad+(\lambda-1) \frac{\left(\frac{1}{\lambda}-1\right) e^{(y-x) v}}{\frac{1}{\lambda} e^{v}-1}\left(\frac{e^{x(u+v)}}{e^{u+v}-1}-\frac{1}{u+v}\right) \\
\quad+\frac{1-\lambda}{u+v}\left(\frac{(\lambda-1) e^{(1+x-y) u}}{\lambda e^{u}-1}-\frac{\left(\frac{1}{\lambda}-1\right) e^{(y-x) v}}{\frac{1}{\lambda} e^{v}-1}\right) .
\end{aligned}
$$

Notice that from (1.4) and the Taylor theorem we have

$$
\begin{aligned}
\frac{e^{x(u+v)}}{e^{u+v}-1}-\frac{1}{u+v} & =\sum_{n=0}^{\infty} \frac{\partial^{n}}{\partial u^{n}}\left(\frac{e^{x u}}{e^{u}-1}-\frac{1}{u}\right) \frac{v^{n}}{n !} \\
& =\sum_{m=0}^{\infty} \sum_{n=0}^{\infty} \frac{B_{m+n+1}(x)}{m+n+1} \cdot \frac{u^{m}}{m !} \cdot \frac{v^{n}}{n !} .
\end{aligned}
$$


On the other hand, if we apply $u^{m}=\sum_{n=0}^{m}\left(\begin{array}{c}m \\ n\end{array}\right)(u+v)^{n}(-v)^{m-n}$ to $(2.5)$, then we get

$$
\begin{aligned}
\frac{(\lambda-1) e^{(1+x-y) u}}{\lambda e^{u}-1}= & \sum_{n=0}^{\infty} \sum_{m=n}^{\infty} \frac{H_{m}\left(1+x-y \mid \frac{1}{\lambda}\right)}{m !}\left(\begin{array}{c}
m \\
n
\end{array}\right)(u+v)^{n}(-v)^{m-n} \\
= & \sum_{n=0}^{\infty} \sum_{m=n+1}^{\infty} \frac{H_{m}\left(1+x-y \mid \frac{1}{\lambda}\right)}{m !}\left(\begin{array}{c}
m \\
n+1
\end{array}\right)(u+v)^{n+1}(-v)^{m-(n+1)} \\
& +\sum_{m=0}^{\infty} H_{m}\left(1+x-y \mid \frac{1}{\lambda}\right) \frac{(-v)^{m}}{m !}
\end{aligned}
$$

Since the Frobenius-Euler polynomials satisfy the symmetric distribution $H_{n}\left(1-x \mid \frac{1}{\lambda}\right)=$ $(-1)^{n} H_{n}(x \mid \lambda)$ for non-negative integer $n$ (see, e.g., [4]), so by (2.5) we can rewrite (2.25) as

$$
\begin{aligned}
\frac{1}{u+v} & \left(\frac{(\lambda-1) e^{(1+x-y) u}}{\lambda e^{u}-1}-\frac{\left(\frac{1}{\lambda}-1\right) e^{(y-x) v}}{\frac{1}{\lambda} e^{v}-1}\right) \\
& =\sum_{n=0}^{\infty} \sum_{m=n+1}^{\infty} \frac{(-1)^{n+1} H_{m}(y-x \mid \lambda)}{m !}\left(\begin{array}{c}
m \\
n+1
\end{array}\right)(u+v)^{n} v^{m-(n+1)} \\
& =\sum_{n=0}^{\infty} \sum_{m=n+1}^{\infty} \frac{(-1)^{n+1} H_{m}(y-x \mid \lambda)}{m !}\left(\begin{array}{c}
m \\
n+1
\end{array}\right) \sum_{k=0}^{n}\left(\begin{array}{l}
n \\
k
\end{array}\right) u^{k} v^{m-(k+1)} \\
& =\sum_{k=0}^{\infty} \sum_{n=k}^{\infty} \frac{\sum_{m=n+1}^{\infty} \frac{(-1)^{n+1} H_{m}(y-x \mid \lambda)}{m !}\left(\begin{array}{c}
m \\
n+1
\end{array}\right)\left(\begin{array}{l}
n \\
k
\end{array}\right) u^{k} v^{m-(k+1)}}{m !} \sum^{\infty} \sum_{k=0}^{\infty} \sum_{m=k+1}^{\infty} \frac{(-1)^{k+1} H_{m}(y-x \mid \lambda)}{m k v^{m-(k+1)}} \\
& =\sum_{k=0}^{\infty} \sum_{m=0}^{\infty} \frac{(-1)^{k+1} k ! \cdot m ! \cdot H_{k+m+1}(y-x \mid \lambda)}{(k+m+1) !} \cdot \frac{u^{k}}{k !} \cdot \frac{v^{m}}{m !} \\
& =\sum_{m=0}^{\infty} \sum_{n=0}^{\infty} \frac{(-1)^{m+1} m ! \cdot n ! \cdot H_{m+n+1}(y-x \mid \lambda)}{(m+n+1) !} \cdot \frac{u^{m}}{m !} \cdot \frac{v^{n}}{n !} .
\end{aligned}
$$

Hence, applying (2.5), (2.24) and (2.26) to (2.23) and then comparing the coefficients of $u^{m} v^{n} / m ! \cdot n !$ gives

$$
\begin{aligned}
H_{m}\left(x \mid \frac{1}{\lambda}\right) H_{n}(y \mid \lambda)= & (1-\lambda) \sum_{k=0}^{m}\left(\begin{array}{c}
m \\
k
\end{array}\right) H_{m-k}\left(1+x-y \mid \frac{1}{\lambda}\right) \frac{B_{n+k+1}(y)}{n+k+1} \\
& +(\lambda-1) \sum_{k=0}^{n}\left(\begin{array}{l}
n \\
k
\end{array}\right) H_{n-k}(y-x \mid \lambda) \frac{B_{m+k+1}(x)}{m+k+1} \\
& +(-1)^{m}(\lambda-1) \frac{m ! \cdot n !}{(m+n+1) !} H_{m+n+1}(y-x \mid \lambda),
\end{aligned}
$$

which together with (2.11) yields the desired result.

It becomes obvious that setting $x=y$ and then substituting $m$ for $n$ and $n$ for $m$ in Theorem 2.3 gives formula (1.8). 
Theorem 2.4 Let $n$ be a non-negative integer. Then, for $\lambda \neq 1, \mu \neq 1, \lambda \mu \neq 1$,

$$
\begin{aligned}
\sum_{k=0}^{n} H_{k}(x \mid \lambda) H_{n-k}(y \mid \mu)= & \frac{\lambda(\mu-1)}{\lambda \mu-1} \sum_{k=0}^{n}\left(\begin{array}{l}
n+1 \\
k+1
\end{array}\right) H_{k}(x-y \mid \lambda) H_{n-k}(y \mid \lambda \mu) \\
& +\frac{\mu(\lambda-1)}{\lambda \mu-1} \sum_{k=0}^{n}\left(\begin{array}{l}
n+1 \\
k+1
\end{array}\right) H_{k}(y-x \mid \mu) H_{n-k}(x \mid \lambda \mu) \\
& -\frac{(\lambda-1)(\mu-1)}{\lambda \mu-1} \sum_{k=0}^{n}\left(\begin{array}{l}
n+1 \\
k+1
\end{array}\right)(x-y)^{k} H_{n-k}(y \mid \lambda \mu) .
\end{aligned}
$$

Proof It is easy to see that (2.2) can be rewritten as

$$
\begin{aligned}
H_{m}(x \mid \lambda) H_{n}(y \mid \mu)= & \frac{\lambda(\mu-1)}{\lambda \mu-1} \sum_{k=0}^{m}\left(\begin{array}{c}
m \\
k
\end{array}\right) H_{k}(x-y \mid \lambda) H_{m+n-k}(y \mid \lambda \mu) \\
& +\frac{\mu(\lambda-1)}{\lambda \mu-1} \sum_{k=0}^{n}\left(\begin{array}{l}
n \\
k
\end{array}\right) H_{k}(y-x \mid \mu) H_{m+n-k}(x \mid \lambda \mu) \\
& -\frac{(\lambda-1)(\mu-1)}{\lambda \mu-1} \sum_{k=0}^{m}\left(\begin{array}{c}
m \\
k
\end{array}\right)(x-y)^{k} H_{m+n-k}(y \mid \lambda \mu) .
\end{aligned}
$$

Hence, by substituting $l$ for $m$ and $n-l$ for $n$ with $0 \leq l \leq n$ and then making the summation operation $\sum_{l=0}^{n}$ in (2.29), we have

$$
\begin{aligned}
\sum_{l=0}^{n} H_{l}(x \mid \lambda) H_{n-l}(y \mid \mu)= & \frac{\lambda(\mu-1)}{\lambda \mu-1} \sum_{l=0}^{n} \sum_{k=0}^{l}\left(\begin{array}{l}
l \\
k
\end{array}\right) H_{k}(x-y \mid \lambda) H_{n-k}(y \mid \lambda \mu) \\
& +\frac{\mu(\lambda-1)}{\lambda \mu-1} \sum_{l=0}^{n} \sum_{k=0}^{l}\left(\begin{array}{l}
l \\
k
\end{array}\right) H_{k}(y-x \mid \mu) H_{n-k}(x \mid \lambda \mu) \\
& -\frac{(\lambda-1)(\mu-1)}{\lambda \mu-1} \sum_{l=0}^{n} \sum_{k=0}^{l}\left(\begin{array}{l}
l \\
k
\end{array}\right)(x-y)^{k} H_{n-k}(y \mid \lambda \mu) .
\end{aligned}
$$

If we change the order of summations on the right-hand side of (2.30), then we obtain

$$
\begin{aligned}
\sum_{l=0}^{n} H_{l}(x \mid \lambda) H_{n-l}(y \mid \mu)= & \frac{\lambda(\mu-1)}{\lambda \mu-1} \sum_{k=0}^{n} \sum_{l=k}^{n}\left(\begin{array}{l}
l \\
k
\end{array}\right) H_{k}(x-y \mid \lambda) H_{n-k}(y \mid \lambda \mu) \\
& +\frac{\mu(\lambda-1)}{\lambda \mu-1} \sum_{k=0}^{n} \sum_{l=k}^{n}\left(\begin{array}{l}
l \\
k
\end{array}\right) H_{k}(y-x \mid \mu) H_{n-k}(x \mid \lambda \mu) \\
& -\frac{(\lambda-1)(\mu-1)}{\lambda \mu-1} \sum_{k=0}^{n} \sum_{l=k}^{n}\left(\begin{array}{l}
l \\
k
\end{array}\right)(x-y)^{k} H_{n-k}(y \mid \lambda \mu) .
\end{aligned}
$$

Notice that for non-negative integers $m, n$ (see, e.g., [27, Lemma 3.1]),

$$
\sum_{k=m}^{n}\left(\begin{array}{l}
k \\
m
\end{array}\right)=\left(\begin{array}{c}
n+1 \\
m+1
\end{array}\right)
$$

Thus, the desired result follows by applying (2.32) to (2.31). 
We next discuss some special cases of Theorem 2.4. By setting $x=y$ in Theorem 2.4, we obtain that for a non-negative integer $n$,

$$
\begin{aligned}
\sum_{k=0}^{n} H_{k}(x \mid \lambda) H_{n-k}(x \mid \mu)= & \frac{\lambda(\mu-1)}{\lambda \mu-1} \sum_{k=0}^{n}\left(\begin{array}{l}
n+1 \\
k+1
\end{array}\right) H_{k}(\lambda) H_{n-k}(x \mid \lambda \mu) \\
& +\frac{\mu(\lambda-1)}{\lambda \mu-1} \sum_{k=0}^{n}\left(\begin{array}{c}
n+1 \\
k+1
\end{array}\right) H_{k}(\mu) H_{n-k}(x \mid \lambda \mu) \\
& -\frac{(\lambda-1)(\mu-1)}{\lambda \mu-1}(n+1) H_{n}(x \mid \lambda \mu) .
\end{aligned}
$$

Taking $\lambda=\mu$ in (2.33) we have

$$
\begin{aligned}
\sum_{k=0}^{n} H_{k}(x \mid \lambda) H_{n-k}(x \mid \lambda)= & \frac{2 \lambda}{\lambda+1} \sum_{k=0}^{n}\left(\begin{array}{l}
n+1 \\
k+1
\end{array}\right) H_{k}(\lambda) H_{n-k}\left(x \mid \lambda^{2}\right) \\
& -\frac{\lambda-1}{\lambda+1}(n+1) H_{n}\left(x \mid \lambda^{2}\right)
\end{aligned}
$$

which is equivalent to

$$
\begin{aligned}
\sum_{k=0}^{n} H_{k}(x \mid \lambda) H_{n-k}(x \mid \lambda)= & \frac{2 \lambda}{\lambda+1} \sum_{k=0}^{n}\left(\begin{array}{c}
n+1 \\
k
\end{array}\right) H_{n-k}(\lambda) H_{k}\left(x \mid \lambda^{2}\right) \\
& -\frac{\lambda-1}{\lambda+1}(n+1) H_{n}\left(x \mid \lambda^{2}\right) .
\end{aligned}
$$

It is interesting to point out that formula (2.35) is analogous to the following sum relation on the classical Bernoulli polynomials:

$$
\sum_{k=0}^{n} B_{k}(x) B_{n-k}(x)-\frac{2}{n+2} \sum_{k=0}^{n-2}\left(\begin{array}{c}
n+2 \\
k
\end{array}\right) B_{n-k} B_{k}(x)=(n+1) B_{n}(x),
$$

which was obtained by Kim et al. [25] using a nice method called the Bernoulli basis $\left\{B_{0}(x), \ldots, B_{n}(x)\right\}$ for $\mathbb{P}_{n}$ consisting of the Bernoulli polynomials with $\mathbb{P}_{n}$ being the space of polynomials of degree less than or equal to $n$. For an equivalent version of (2.35), see [9, Theorem 3] for details.

Theorem 2.5 Let $n \geq 2$ be a positive integer. Then, for $\lambda \neq 1, \mu \neq 1, \lambda \mu \neq 1$,

$$
\begin{aligned}
\sum_{k=1}^{n-1} \frac{H_{k}(x \mid \lambda) H_{n-k}(y \mid \mu)}{k(n-k)} & \\
= & \frac{1}{n} \sum_{k=1}^{n-1}\left(\begin{array}{l}
n \\
k
\end{array}\right)\left(H_{n-1}-H_{k-1}\right)\left\{\frac{\lambda(\mu-1)}{\lambda \mu-1} H_{k}(x-y \mid \lambda) H_{n-k}(y \mid \lambda \mu)\right. \\
& +\frac{\mu(\lambda-1)}{\lambda \mu-1} H_{k}(y-x \mid \mu) H_{n-k}(x \mid \lambda \mu) \\
& \left.-\frac{(\lambda-1)(\mu-1)}{\lambda \mu-1}(x-y)^{k} H_{n-k}(y \mid \lambda \mu)\right\}
\end{aligned}
$$




$$
\begin{aligned}
& +2 H_{n-1}\left(\frac{\lambda(\mu-1)}{\lambda \mu-1} \cdot \frac{H_{n}(y \mid \lambda \mu)}{n}+\frac{\mu(\lambda-1)}{\lambda \mu-1} \cdot \frac{H_{n}(x \mid \lambda \mu)}{n}\right. \\
& \left.-\frac{(\lambda-1)(\mu-1)}{\lambda \mu-1} \cdot \frac{H_{n}(y \mid \lambda \mu)}{n}\right) .
\end{aligned}
$$

Proof Let $m, n$ be positive integers. Then, by multiplying $1 / m n$ on both sides of (2.29) and then substituting $l$ for $m$ and $n-l$ for $n$ with $1 \leq l \leq n-1$, in light of making the summation operation $\sum_{l=1}^{n-1}$, we get

$$
\begin{aligned}
\sum_{l=1}^{n-1} & \frac{H_{l}(x \mid \lambda) H_{n-l}(y \mid \mu)}{l(n-l)} \\
= & \frac{\lambda(\mu-1)}{\lambda \mu-1} \sum_{l=1}^{n-1} \frac{1}{l(n-l)} \sum_{k=0}^{l}\left(\begin{array}{l}
l \\
k
\end{array}\right) H_{k}(x-y \mid \lambda) H_{n-k}(y \mid \lambda \mu) \\
& +\frac{\mu(\lambda-1)}{\lambda \mu-1} \sum_{l=1}^{n-1} \frac{1}{l(n-l)} \sum_{k=0}^{l}\left(\begin{array}{l}
l \\
k
\end{array}\right) H_{k}(y-x \mid \mu) H_{n-k}(x \mid \lambda \mu) \\
& -\frac{(\lambda-1)(\mu-1)}{\lambda \mu-1} \sum_{l=1}^{n-1} \frac{1}{l(n-l)} \sum_{k=0}^{l}\left(\begin{array}{l}
l \\
k
\end{array}\right)(x-y)^{k} H_{n-k}(y \mid \lambda \mu) .
\end{aligned}
$$

Observe that for positive integers $m, n$,

$$
\frac{1}{m n}=\frac{1}{m+n}\left(\frac{1}{m}+\frac{1}{n}\right)
$$

Hence, by applying (2.39) to (2.38), we obtain

$$
\begin{aligned}
\sum_{l=1}^{n-1} & \frac{H_{l}(x \mid \lambda) H_{n-l}(y \mid \mu)}{l(n-l)} \\
= & \frac{\lambda(\mu-1)}{\lambda \mu-1} \cdot \frac{1}{n} \sum_{l=1}^{n-1}\left(\frac{1}{l}+\frac{1}{n-l}\right) \sum_{k=1}^{l}\left(\begin{array}{l}
l \\
k
\end{array}\right) H_{k}(x-y \mid \lambda) H_{n-k}(y \mid \lambda \mu) \\
& +\frac{\mu(\lambda-1)}{\lambda \mu-1} \cdot \frac{1}{n} \sum_{l=1}^{n-1}\left(\frac{1}{l}+\frac{1}{n-l}\right) \sum_{k=1}^{l}\left(\begin{array}{l}
l \\
k
\end{array}\right) H_{k}(y-x \mid \mu) H_{n-k}(x \mid \lambda \mu) \\
& -\frac{(\lambda-1)(\mu-1)}{\lambda \mu-1} \cdot \frac{1}{n} \sum_{l=1}^{n-1}\left(\frac{1}{l}+\frac{1}{n-l}\right) \sum_{k=1}^{l}\left(\begin{array}{l}
l \\
k
\end{array}\right)(x-y)^{k} H_{n-k}(y \mid \lambda \mu) \\
& +2 H_{n-1}\left(\frac{\lambda(\mu-1)}{\lambda \mu-1} \cdot \frac{H_{n}(y \mid \lambda \mu)}{n}\right. \\
& +\frac{\mu(\lambda-1)}{\lambda \mu-1} \cdot \frac{H_{n}(x \mid \lambda \mu)}{n} \\
& \left.-\frac{(\lambda-1)(\mu-1)}{\lambda \mu-1} \cdot \frac{H_{n}(y \mid \lambda \mu)}{n}\right) .
\end{aligned}
$$


Changing the order of summations on the right-hand side of (2.40), we obtain

$$
\begin{aligned}
\sum_{l=1}^{n-1} \frac{H_{l}(x \mid \lambda) H_{n-l}(y \mid \mu)}{l(n-l)} \\
=\frac{\lambda(\mu-1)}{\lambda \mu-1} \cdot \frac{1}{n} \sum_{k=1}^{n-1} \sum_{l=k}^{n-1}\left(\begin{array}{l}
l \\
k
\end{array}\right)\left(\frac{1}{l}+\frac{1}{n-l}\right) H_{k}(x-y \mid \lambda) H_{n-k}(y \mid \lambda \mu) \\
\quad+\frac{\mu(\lambda-1)}{\lambda \mu-1} \cdot \frac{1}{n} \sum_{k=1}^{n-1} \sum_{l=k}^{n-1}\left(\begin{array}{l}
l \\
k
\end{array}\right)\left(\frac{1}{l}+\frac{1}{n-l}\right) H_{k}(y-x \mid \mu) H_{n-k}(x \mid \lambda \mu) \\
\quad-\frac{(\lambda-1)(\mu-1)}{\lambda \mu-1} \cdot \frac{1}{n} \sum_{k=1}^{n-1} \sum_{l=k}^{n-1}\left(\begin{array}{l}
l \\
k
\end{array}\right)\left(\frac{1}{l}+\frac{1}{n-l}\right)(x-y)^{k} H_{n-k}(y \mid \lambda \mu) \\
\quad+2 H_{n-1}\left(\frac{\lambda(\mu-1)}{\lambda \mu-1} \cdot \frac{H_{n}(y \mid \lambda \mu)}{n}+\frac{\mu(\lambda-1)}{\lambda \mu-1} \cdot \frac{H_{n}(x \mid \lambda \mu)}{n}\right. \\
\left.\quad-\frac{(\lambda-1)(\mu-1)}{\lambda \mu-1} \cdot \frac{H_{n}(y \mid \lambda \mu)}{n}\right) .
\end{aligned}
$$

Note that for non-negative integers $m, n$ (see, e.g., [27, Lemma 3.1]),

$$
\sum_{k=m}^{n}\left(\begin{array}{c}
k \\
m
\end{array}\right) \frac{1}{k}=\frac{1}{m} \sum_{k=m}^{n}\left(\begin{array}{c}
k-1 \\
m-1
\end{array}\right)=\frac{1}{m}\left(\begin{array}{c}
n \\
m
\end{array}\right)
$$

and

$$
\sum_{k=m}^{n}\left(\begin{array}{l}
k \\
m
\end{array}\right) \frac{1}{n+1-k}=\left(H_{n+1}-H_{m}\right)\left(\begin{array}{c}
n+1 \\
m
\end{array}\right)
$$

By combining (2.42) and (2.43), we have

$$
\begin{aligned}
\sum_{k=m}^{n}\left(\begin{array}{c}
k \\
m
\end{array}\right)\left(\frac{1}{k}+\frac{1}{n+1-k}\right) & =\frac{1}{m}\left(\begin{array}{c}
n \\
m
\end{array}\right)+\left(H_{n+1}-H_{m}\right)\left(\begin{array}{c}
n+1 \\
m
\end{array}\right) \\
& =\left(H_{n}-H_{m-1}\right)\left(\begin{array}{c}
n+1 \\
m
\end{array}\right) .
\end{aligned}
$$

Thus, the desired result follows by applying (2.44) to (2.41).

Obviously the case $x=y$ and $\lambda=\mu$ in Theorem 2.5 gives that for positive integer $n \geq 2$ and $\lambda \neq \pm 1$,

$$
\begin{aligned}
\sum_{k=1}^{n-1} \frac{H_{k}(x \mid \lambda) H_{n-k}(x \mid \lambda)}{k(n-k)}= & \frac{\lambda}{\lambda+1} \cdot \frac{2}{n} \sum_{k=1}^{n-1}\left(\begin{array}{l}
n \\
k
\end{array}\right)\left(H_{n-1}-H_{k-1}\right) H_{k}(\lambda) H_{n-k}\left(x \mid \lambda^{2}\right) \\
& +2 H_{n-1} \frac{H_{n}\left(x \mid \lambda^{2}\right)}{n},
\end{aligned}
$$

which is analogous to the sum relation on the classical Euler polynomials stated in [25, Theorem 7]. From the above, one can also use Theorems 2.2 and 2.3 to establish some 
similar sum relations to Theorems 2.4 and 2.5. We leave the details to the interested readers for an exercise.

\section{Competing interests}

The authors declare that they have no competing interests.

\section{Authors' contributions}

All authors contributed equally to the writing of this paper. All authors read and approved the final manuscript.

\section{Acknowledgements}

This work is supported by the Foundation for Fostering Talents in Kunming University of Science and Technology (Grant No. KKSY201307047) and the National Natural Science Foundation of P.R. China (Grant No. 11326050, 11071194).

Received: 12 April 2014 Accepted: 14 June 2014 Published: 22 Jul 2014

\section{References}

1. Frobenius, FG: Über die Bernoullischen Zahlen und die Eulerischen Polynome. Sitzungsber. K. Preußischen Akad. Wissenschaft. Berlin, pp. 809-847. (1910)

2. Araci, S, Acikgoz, M: A note on the Frobenius-Euler numbers and polynomials associated with Bernstein polynomials. Adv. Stud. Contemp. Math. 22, 399-406 (2012)

3. Araci, S, Şen, E, Acikgoz, M: Theorems on Genocchi polynomials of higher order arising from Genocchi basis. Taiwan. J. Math. 18, 473-482 (2014)

4. Choi, J, Kim, DS, Kim, T, Kim, YH: A note on some identities of Frobenius-Euler numbers and polynomials. Int. J. Math. Math. Sci. 2012, Article ID 861797 (2012)

5. Kim, T, Lee, B: Some identities of the Frobenius-Euler polynomials. Abstr. Appl. Anal. 2009, Article ID 639439 (2009)

6. Kim, T: An identity of the symmetry for the Frobenius-Euler polynomials associated with the fermionic $p$-adic invariant $q$-integrals on $\mathbb{Z}_{p}$. Rocky Mt. J. Math. 41, 239-247 (2011)

7. Kim, T, Choi, J: A note on the product of Frobenius-Euler polynomials arising from the $p$-adic integral on $\mathbb{Z}_{p}$. Adv. Stud. Contemp. Math. 22, 215-223 (2012)

8. Kim, T: Identities involving Frobenius-Euler polynomials arising from non-linear differential equations. J. Number Theory 132, 2854-2865 (2012)

9. Kim, DS, Kim, T: Some new identities of Frobenius-Euler numbers and polynomials. J. Inequal. Appl. 2012, Article ID $307(2012)$

10. Kim, DS, Kim, T, Lee, S-H, Rim, S-H: Frobenius-Euler polynomials and umbral calculus in the $p$-adic case. Adv. Differ Equ. 2012, Article ID 222 (2012)

11. Kim, DS, Kim, T, Lee, S-H: Umbral calculus and the Frobenius-Euler polynomials. Abstr. Appl. Anal. 2013, Article ID $871512(2013)$

12. Simsek, Y, Bayad, A, Lokesha, V: $q$-Bernstein polynomials related to $q$-Frobenius-Euler polynomials, I-functions, and q-Stirling numbers. Math. Methods Appl. Sci. 35, 877-884 (2012)

13. Simsek, Y: Generating functions for generalized Stirling type numbers, array type polynomials, Eulerian type polynomials and their applications. Fixed Point Theory Appl. 2013, Article ID 87 (2013)

14. Cohen, H: Number Theory, Volume II, Analytic and Modern Tools. Graduate Texts in Math., vol. 240. Springer, New York (2007)

15. Nielsen, N: Traité élémentaire des nombres de Bernoulli. Gauthier-Villars, Paris (1923)

16. Nörlund, NE: Vorlesungen über Differenzenrechnung. Springer, Berlin (1924)

17. Srivastava, HM, Choi, J: Zeta and q-Zeta Functions and Associated Series and Integrals. Elsevier, Amsterdam (2012)

18. Carlitz, L: Note on the integral of the product of several Bernoulli polynomials. J. Lond. Math. Soc. 34, 361-363 (1959)

19. He, Y, Wang, CP: Some formulae of products of the Apostol-Bernoulli and Apostol-Euler polynomials. Discrete Dyn. Nat. Soc. 2012, Article ID 927953 (2012)

20. He, Y, Wang, CP: Recurrence formulae for Apostol-Bernoulli and Apostol-Euler polynomials. Adv. Differ. Equ. 2012, Article ID 209 (2012)

21. He, Y, Zhang, WP: A convolution formula for Bernoulli polynomials. Ars Comb. 108, 97-104 (2013)

22. He, Y, Zhang, WP: A three-term reciprocity formula for Bernoulli polynomials. Util. Math. (to appear)

23. Wang, JZ: New recurrence formulae for the Apostol-Bernoulli and Apostol-Euler polynomials. Adv. Differ. Equ. 2013, Article ID 247 (2013)

24. Carlitz, L: The product of two Eulerian polynomials. Math. Mag. 36, 37-41 (1963)

25. Kim, DS, Kim, T, Lee, S-H, Kim, Y-H: Some identities for the product of two Bernoulli and Euler polynomials. Adv. Differ. Equ. 2012, Article ID 95 (2012)

26. Abramowitz, M, Stegun, IA: Handbook of Mathematical Functions with Formulas Graphs and Mathematical Tables. National Bureau of Standards, Washington, DC (1964)

27. He, Y, Zhang, WP: Some sum relations involving Bernoulli and Euler polynomials. Integral Transforms Spec. Funct. 22, 207-215 (2011)

10.1186/1029-242X-2014-261

Cite this article as: He and Wang: New formulae of products of the Frobenius-Euler polynomials. Journal of Inequalities and Applications 2014, 2014:261 